\title{
Dinoflagellate bioluminescence in response to mechanical stimuli in water flows
}

\author{
A. S. Cussatlegras ${ }^{1}$ and P. Le Gal ${ }^{2}$ \\ ${ }^{1}$ DIMAR, UMR 6540 CNRS-Université de la Méditerranée, Centre d'Océanologie de Marseille, Parc Scientifique et \\ technologique de Luminy, Case 901, 13288 Marseille cedex 9, France \\ ${ }^{2}$ IRPHE, UMR 6594 CNRS-Universités d'Aix-Marseille I \& II, 49 rue F. Joliot-curie, BP 146, 13384 Marseille cedex 13, \\ France
}

Received: 19 October 2004 - Revised: 2 December 2004 - Accepted: 6 December 2004 - Published: 21 February 2005

\begin{abstract}
Bioluminescence of plankton organisms induced by water movements has long been observed and is still under investigations because of its great complexity. In particular, the exact mechanism occurring at the level of the cell has not been yet fully understood. This work is devoted to the study of the bioluminescence of the dinoflagellates plankton species Pyrocystis noctiluca in response to mechanical stimuli generated by water flows. Several experiments were performed with different types of flows in a Couette shearing apparatus. All of them converge to the conclusion that stationary homogeneous laminar shear does not trigger massive bioluminescence, but that acceleration and shear are both necessary to stimulate together an intense bioluminescence response. The distribution of the experimental bioluminescence thresholds is finally calculated from the light emission response for the Pyrocystis noctiluca species.
\end{abstract}

\section{Introduction}

Bioluminescence, which is the emission of light by living organisms, is very common in the marine environment, but among phytoplankton, only some species of dinoflagellates are bioluminescent. In these organisms, the light emission is triggered by a mechanical stimulation due to water agitation that induces cell deformations which in turn activate a vacuole membrane action potential. Consequently the entry of hydrogen ions into some sub-cellular organelles called scintillons is possible (Fogel et al., 1972). Then a series of biochemical reactions involving the catalytic oxidation of a specific photo-protein (luciferin) releases some energy in the form of blue light flashes.

In a seminal article (Rohr et al., 1997), the use of bioluminescence of phytoplankton as a flow diagnostic was for the first time evoked. In particular, these authors showed that there was a relation between the intensity of the emit-

Correspondence to: $\mathrm{P}$. Le Gal

(legal@irphe.univ-mrs.fr) ted light and the wall shear stress produced by laminar and turbulent pipe flows. Thus, it could be possible to get quantitative measurements of flow fields if the bioluminescent response was clearly scaled versus the flow parameters. Visualization of the flow patterns around dolphins, in the wakes of spheres (Rohr et al., 1998) or more recently in breaking waves (Stokes et al., 2004) are certainly today the most achieved tentative towards a quantitative use of bioluminescence as a tool to probe laminar and turbulent flows. Figure 1 presents another example that we obtained in a fully developed turbulent flow. These images show a turbulent field created by the contra-rotation of two facing propellers in a cubic box. This kind of flow, called the von Karman flow, has already been used to study different properties of turbulence (Cadot et al., 1995; Danaila et al., 1999). In this experiment, the fluid is sea water seeded with a culture of dinoflagellates. As seen on these images, the light intensity emitted by the plankton cells varies in time and in space. Therefore, it should be in principle possible to get quantitative measurements of the turbulent flow by image analysis of the emitted light in function of space and time. This technique would then improve a lot the traditional single point measurements performed by hot wires (only one or few spatial positions) or the more recently used particle image velocimetry (extended measurement in space but limited temporal resolution, except for the use of fast pulsed Lasers and high resolution cameras).

In a former study, Latz et al. (1994), have showed that the emitted light intensity follows power laws of the laminar fluid shears associated with simple Couette flows. These flows are created between two concentric differentially rotating cylinders. Well-defined thresholds between 0.1 and $0.3 \mathrm{~N} \mathrm{~m}^{-2}$ of the applied shear were determined for different species of dinoflagellates. Revisiting this Couette flow experiment, Cussatlegras and Le Gal (2004), showed that contrary to the observations of Latz et al. (1994), a pure laminar stationary and homogenous shear flow does not excite the main bioluminescent response in Pyrocystis noctiluca. The bulk of bioluminescence emissions primarily occurred under 

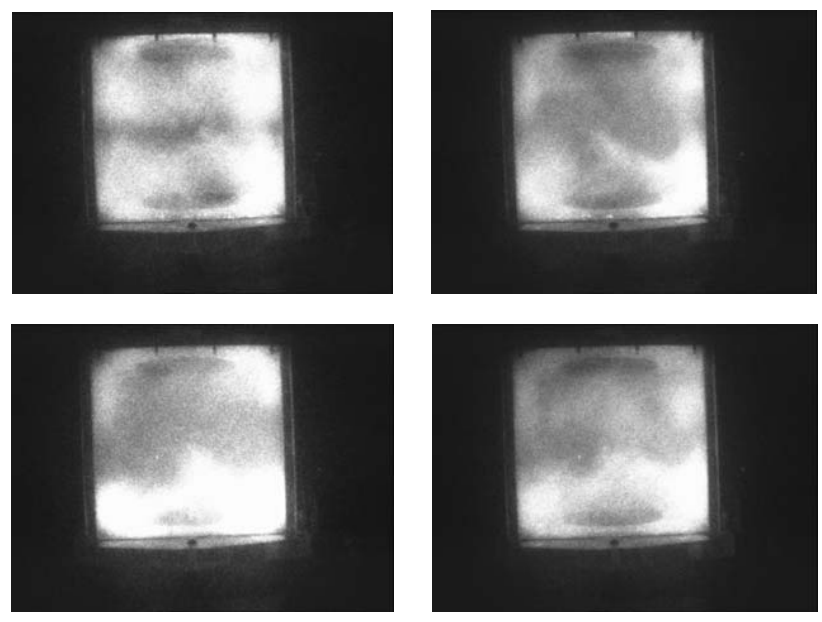

Fig. 1. Four different snapshots of the turbulent von Karman flow seeded with dinoflagellate cells. The Karman flow is created by two rotating facing propellers. The intense shear created by the counterrotation of these propellers destabilizes the whole flow that becomes fully turbulent.

non homogenous or non stationary flow conditions, where the cells experience shear changes as they are advected by the flow. In particular, the transition to turbulence triggers a strong light emission. These findings show the crucial role of shear flow acceleration and agree with the results of Blaser et al. (2002).

Blaser et al. (2002) revisited also previous experiments realized with pipe flows (Latz and Rohr, 1999; Rohr et al. 2002). They note in particular that the bioluminescent threshold they measure is higher $\left(0.6 \mathrm{Nm}^{-2}\right)$ than the threshold obtained from the previous experiments. Moreover they show that if bioluminescence is possible in laminar flow, it is observed for occasionnal flashes or at the pipe inlet or when an aperture or a curvature affects the flow. They also report a large increase of bioluminescence when transition to turbulence occcurs (see also Rohr et al., 1990). As we will see later, these observations agree with our present findings. Our interpretation of this pipe flow bioluminescence excitation is that the dinoflagellate cells travel through the nozzle of the pipe and feel changes in flow conditions on a length corresponding to the entrance length of the pipe. In the Lagrangian frame of reference linked to each cell, this is similar to temporal flow variations.

In the present study, three types of experimental configurations have been used to improve the understanding of bioluminescence in dinoflagellates. Three different and new experiments have been realized and complete a work recently published (Cussatlegras and Le Gal, 2004). First, using a Couette apparatus with a smaller gap than in our first experiment (Cussatlegras and Le Gal, 2004), we emphasis the effect of a strong non homogeneity on the laminar shear flow that consequently triggers a higher bioluminescent response. Then, in a second series of experiments, we test the effect of acceleration by abrupt starts of the flow in the absence of shear. This was achieved by co-rotation onsets of both cylinders and the introduction of an inner wall in the gap between them. Finally, the combination of both acceleration and shear is investigated through "simple" (no inner cylinder rotation) or counter-rotation Couette flow experiments. The accelerated flows that we consider here, are created by the abrupt start of one or two of the cylinders of the Couette apparatus. Note that the centripetal acceleration of this rotating Couette flows is always balanced by the radial pressure gradient and is inefficient to stimulate bioluminescence. Finally, we present the derivation of the bioluminescence response that leads to the statistics of light emission under given shear and acceleration in Pyrocystis noctiluca. To the best of our knowledge, this result that gives the probability to trigger bioluminescence from a population of Pyrocystis noctiluca under acceleration and shear is a totally new finding and is of great importance in the attempt to use bioluminescence as a flow diagnostic.

\section{Material and methods}

\subsection{Cultures}

Cultured cells of Pyrocystis noctiluca (Murray and Haeckel) were obtained from the CCMP in Bigelow, Maine (strain number 32). Cultures were maintained at $20^{\circ} \mathrm{C}+/-2^{\circ} \mathrm{C}$ under a $12 \mathrm{~h}$ light: dark cycle using cool-white fluorescent illumination providing approximately $10 \mathrm{~W} \mathrm{~m}^{2}$. Semi-continuous batch cultures were grown in enriched $\mathrm{f} / 2$ media (Guillard and Ryther, 1962). Cultures used for experiments were in exponential growth phase and the concentration has been estimated by counting fixed samples in Nageotte slides under a dissecting microscope. Cell concentrations used for the experiments were $200-1000 \mathrm{cell} \mathrm{ml}^{-1}$ with an accuracy of $10 \%$.

\subsection{Couette device}

The Couette apparatus (Couette, 1890) consists in two coaxial rotating cylinders (Fig. 2) and is fully described in Cussatlegras and Le Gal (2004). It has been designed following Latz et al. (1994). Its total length is $190 \mathrm{~mm}$. The inner diameter of the outer cylinder is $52.3 \mathrm{~mm}$ and the diameter of the inner cylinder is respectively $47.8 \mathrm{~mm}$ in the first experiments (small gap) and $46.3 \mathrm{~mm}$ for the second and third set of experiments. Rotational speed is measured by an optical encoder mounted directly on the rotation axis of the external cylinder of the Couette device. The inner cylinder is of black polished acetal plastic and the outer one is of transparent glass so the light can be measured by an intensified video camera (ULL509, Lhéritier) and a photomultiplier tube (Hamamatsu H6180-01). Taking into account the distance between the Couette chamber and the photomultiplier tube, a correction factor is applied on the light measurement which is then given in number of photons per second and per cell $\left(\mathrm{ph} \mathrm{s}^{-1}\right.$ cell $\left.^{-1}\right)$. 


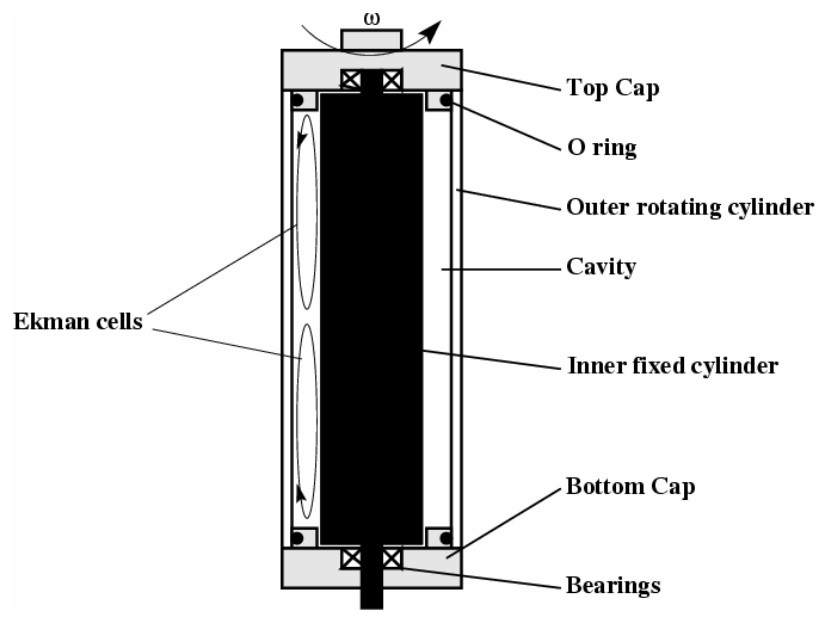

Fig. 2. Schematic diagram of the Couette device that consists in two coaxial cylinders differentially rotating. The total length of the cylinders is $190 \mathrm{~mm}$; the inner diameter of the outer glass cylinder is $52.3 \mathrm{~mm}$ and the gap is $2.25 \mathrm{~mm}$ for the experiments of Sect. 3.1 and $3 \mathrm{~mm}$ for the experiments of Sects. 3.2 and 3.3. The secondary flows due to the presence of Ekman cells have been represented on the left side of the diagram.

Before each experiment, fresh samples were transferred in the dark experimental room. The Couette chamber is filled with a dinoflagellate culture sample by pouring it directly in the space between the cylinder. The experimental runs are started several minutes later in order to let the flow settle down.

Two different protocols have been used. In the first set of experiments, the speed rotation is increased progressively in order to test the effect of shear without acceleration (which is in fact very weak and thus can be neglected). In the second set of experiment, we test abrupt starts of both cylinders rotating in the same direction with a wall placed between them and rotating with them. These runs allow to study the effect of acceleration without shear. Finally, the last set of experiments presents the bioluminescence response to the combination of both shear and acceleration.

\section{Results and discussion}

3.1 Bioluminescence response under shear without acceleration

The experimental protocol for this run is the same as that used in Cussatlegras and Le Gal (2004). The Couette apparatus has a $2.25 \mathrm{~mm}$ gap this time (and not $3 \mathrm{~mm}$ as before) and is driven from outside the dark measurement room. The rotation frequency $\omega$ of the outer cylinder is slowly and continuously increased form 0 to $18 \mathrm{~Hz}$ in $150 \mathrm{~s}$. The inner cylinder is kept at rest. This "quasi-static" ramp which is presented in Fig. 3A avoids any effective acceleration as its value is negligible $\left(0.03 \mathrm{~m} \mathrm{~s}^{-2}\right.$ at the inner boundary of the outer wall). As we will see later, acceleration of shear flows are efficient for bioluminecence only for values two orders of magnitude larger. The totality of the experimental run is video-taped while the light emission is recorded by the photomultiplier. The main trends of the recorded light intensity curve are illustrated by corresponding flow images. Similarly to our previous observations the bioluminescence emission presents three main characteristics (Fig. 3B). The first bell shape pattern is due to the excitation of the plankton cells near the end caps. We believe that this light emission is triggered by the complex flow induced near the singular boundary condition imposed at the corner between the rotating end caps and the stationary inner cylinder. Then, as the excited cells become exhausted, the light emission decreases in correspondence. Note that during this process, several isolated flashes, that may be due to extremely sensitive cells (that may hit the wall for instance), are also visible.

As it was the case in our first study (Cussatlegras and Le Gal, 2004), the rotating caps generate also Ekman cells that consist in two re-circulation eddies entrained by centrifugal effect. This effect is a general feature of experimental Couette flows and is not an artefact of our own device. But in the present case, and contrary to our first observations, the effect of these secondary re-circulation eddies is quite strong because of the gap reduction (more intense gradients) and we can clearly measure this time the light emission associated with the stagnation line created between them in the equatorial region. The stimulation of the cells in the region where the Ekman eddies meet can be explained by the fact that the cells encounter various shear conditions as they travel in the flow. In particular, when arriving in this equatorial region, they abruptly change the direction of their axial velocity. Then bioluminescence decreases as the dinoflagellates in the middle region of the container become exhausted.

Contrary to our first experiments (Cussatlegras and Le Gal, 2004), this small gap experiment allows to measure the axial length of this luminous region (compared to the total cylinder height) which is plotted as a function of the rotational frequency $\omega$ is compatible with a square root behaviour (Fig. 4). Up to now, we have not been able to relate exactly this behaviour with models of the Ekman secondary flow eddies. Note however that this square root behavior is similar to the evolution of the axial velocity of Ekman eddies (see Schilichting, 1970). Nevertheless, it is clear on this figure that non homogeneity leads to bioluminescent excitation of the plankton cells even in the case of laminar stationary flows.

Therefore, we think that in this experiment, the Pyrocystis noctiluca cells encounter various shear flow conditions as they are advected by the main rotating flow plus the secondary eddies. Thus, it appears that temporal changes in the cell environment, seen in the cell's Lagrangian frame of reference, are necessary to trigger the bioluminescent response. Indeed, this is also the case when a sharp peak related to the transition to turbulence of the flow appears after $115 \mathrm{~s}$ (Fig. 3B). At this point, a large amount of the cell population is excited by the chaotic non stationary turbulent field that takes the shape of the well known turbulent spiral, which is 

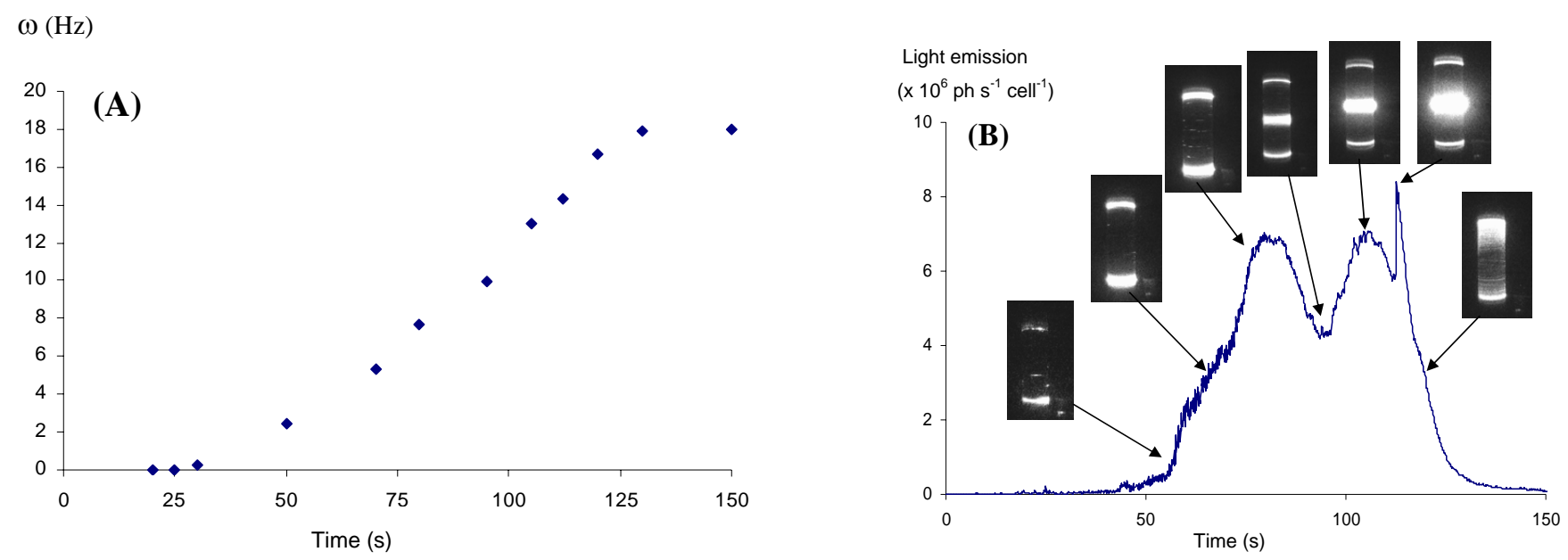

Fig. 3. (A) Evolution of the rotating velocity $\omega$ the Couette device (small gap) versus time during the "quasi-static" ramp. (B) Light emission during this ramp and corresponding images of the flow. Except from rare isolated flashes, no bioluminescence is stimulated in the bulk of the laminar shear flow. The three main qualitative characteristics of the bioluminescence response are in this case: permanent luminous extremities, light emission in the equatorial plane due to Ekman re-circulation eddies (around 100 s) and a sharp peak of light emission corresponding to the onset of turbulence $(115 \mathrm{~s})$ where the shape of the turbulent spiral can be guessed.

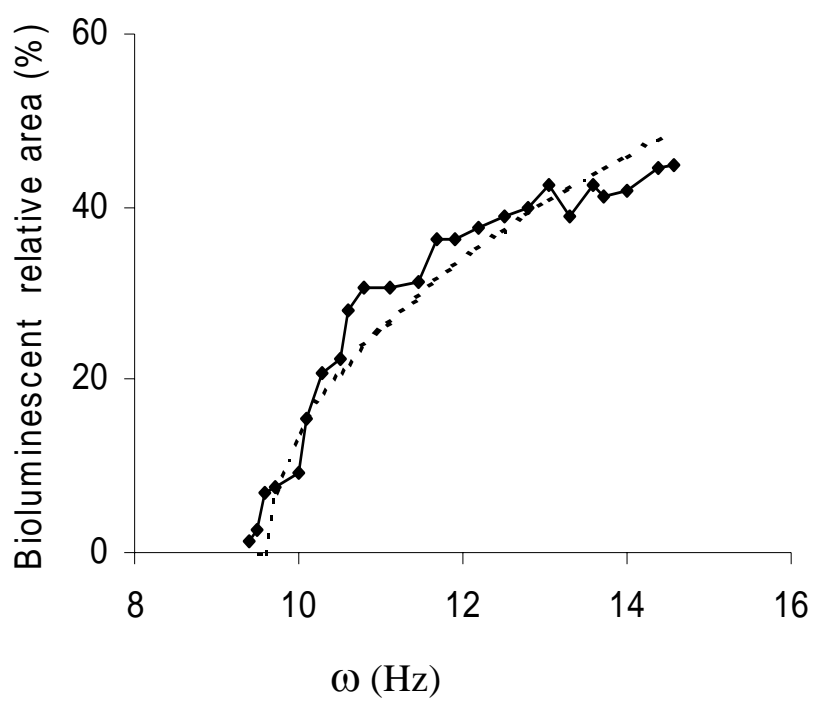

Fig. 4. Relative width of the bioluminescent region corresponding to the stagnation zone between the Ekman re-circulation eddies. The solid curve represents the experimental data points and the dotted curve is a square root fit.

clearly visible on the video recordings. Some trends of this spiral can be guessed on the two last mages of Fig. 3B, where strong non homogeneous patterns are visible.

\subsection{Bioluminescence response under acceleration without shear}

Temporal changes in flows can also be directly created by accelerations of the flow field. In order to test the response of the dinoflagellates to acceleration, we performed two series of experiments. In the first one, where the shear is maintained for further comparison with the second run (no shear), the acceleration is generated by abrupt starts of the outer cylinder of the Couette apparatus. As the flow is set in rotation with a constant acceleration equal to $11.5 \mathrm{~m} \mathrm{~s}^{-2}$ at the outer cylinder wall, a strong non stationary shear is generated in the gap as the inner cylinder is kept stationary.

Light emission and flow images are presented in Fig. 5. These runs correspond to a constant acceleration ramp of the outer cylinder that rotates from 0 to $9 \mathrm{~Hz}$. The solid curve indicates the bioluminescence response versus time and is associated to a global excitation of the dinoflagellate culture as presented by the flow image. In the second experimental run, a vertical thin wall is placed in the gap between the cylinders ( $3 \mathrm{~mm}$ in this case), thus the whole bulk of fluid will be entrained since both cylinders and the wall are set into rotation together at the same rate. In this second run, no shear is created excepted nearby the end caps (we could not avoid the presence of a small gap between the vertical wall and the end caps; this region of the flow is subjected to an Ekman circulation again). As illustrated by the dotted curve response, the intensity of the light emission is reduced: the corresponding visualisation of the flow clearly shows that the cells present in the bulk are not excited. Therefore, we have demonstrated here that acceleration alone does not trigger the bioluminescence response in Pyrocystis noctiluca. This is in fact not very surprising as the density of the plankton cells is very close to that of water and consequently their effective mass is nearly zero. This result is in accordance with Latz et al. (2004), where the effect of shear and acceleration in a nozzle flow was clearly identified. 


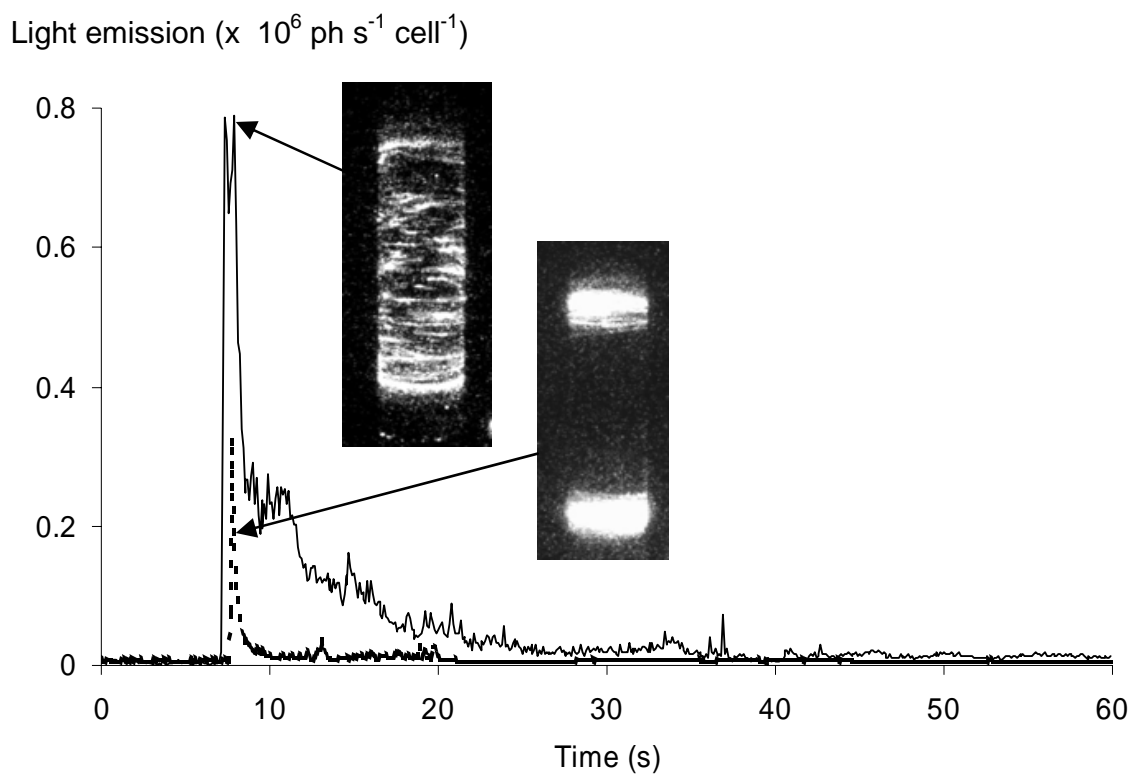

Fig. 5. Bioluminescence response versus time following an abrupt start of the Couette chamber. The final rotation frequency is set to $9 \mathrm{~Hz}$ and the acceleration of the outer cylinder surface to $11.5 \mathrm{~m} \mathrm{~s}^{-2}$. A strong light emission is recorded at the onset. In standard condition, where the cells experience acceleration and shear $\left({ }^{--}\right)$, a massive response in the whole illuminated cylinder is recorded. When only acceleration is applied when both cylinders rotate together and a thin wall is inserted between them (-), the bioluminescence response is weaker and is only stimulated at both end caps. In the bulk, no bioluminescence is stimulated by acceleration alone.

\subsection{Bioluminescence response under shear and accelera- tion}

As seen in the previous section, acceleration alone is ineffective to get the main response of Pyrocystis noctiluca when stimulated by mechanical constraints. However a combination of acceleration and shear (first run of Sect. 3.2) excites bioluminescence. It corresponds in fact to the existence of a shearing velocity whose role has already been studied in various experiments such as the growth of muscle cells (Burkholder, 2003) or the re-orientation of endothelial cells when subjected to cycling forcing (Wang et al., 2001). Physically, this means that the double derivative in time and in space of the velocity field (the shearing velocity) must not be zero to get bioluminescence. This final section is then devoted to the study of bioluminescence during abrupt start of the Couette apparatus with a given acceleration and with shear. Two sets of experiments were realised in which the inner cylinder is respectively at rest or in exact counterrotation. In this last case, it can be easily demonstrated that the mean (in space) shear produced during the transients is always twice that produced when only the outer cylinder rotates (see later). These experiments are labelled "simple" in the case of the simple Couette flow (with no rotation of the inner cylinder) and "counter" when both cylinders are in counter-rotation (Fig. 6). In both cases, the Couette flow is entirely luminous as seen in Fig. 5 (solid line) for relatively short periods of time (typicaly $10 \mathrm{~s}$ or less) when the flow is still accelerating. For small $\omega$ jumps, the flow stays stationnary but this is not the case for the largest $\omega \mathrm{g}$ increments where turbulence can be observed. In fact, the first peak in the bioluminescence response is higher than the following flashes and its intensity is the only quantity that will be kept to plot Fig. 6. This was explained and illustrated in Cussatlegras and Le Gal (2004). It is worth noting that no bioluminescence is triggered after these short periods of time, that is when the shear has reached a constant value.

Both response curves (Fig. 6A) have a sigmoid shape but as it is expected, the plateau where all the cells are excited is reached at a lower rotation frequency for the "counter" experiments because the shear is higher. Both curves represent the same physical response of the dinoflagellate population to the same mechanical stimulation. Thus, these responses should be a unique function of a unique variable. It appears that if we define the mean shear by an integration over the gap of the shear reached at the end of the acceleration ramps (that is simply the difference in cylinder velocities divided by the gap thickness), the shear met in the "counter" experiments is double the shear of the "simple" experiments. Note that this property is always true, even during the transitory flow. When representing the response curves as functions of this maximum mean shear (reached here at the end of the acceleration ramp), it is then clearly visible that experimental data points collapse on a unique curve (Fig. 6B). This means also that the local properties of the flow near the walls during the transients are not determinant for the bioluminescent response. If the contrary was true, twice the volume of cells would be excited in the "counter" experiments during the first instants of the ramps and Fig. 6A clearly shows that this is not the case since twice the quantity of light is not emitted. 

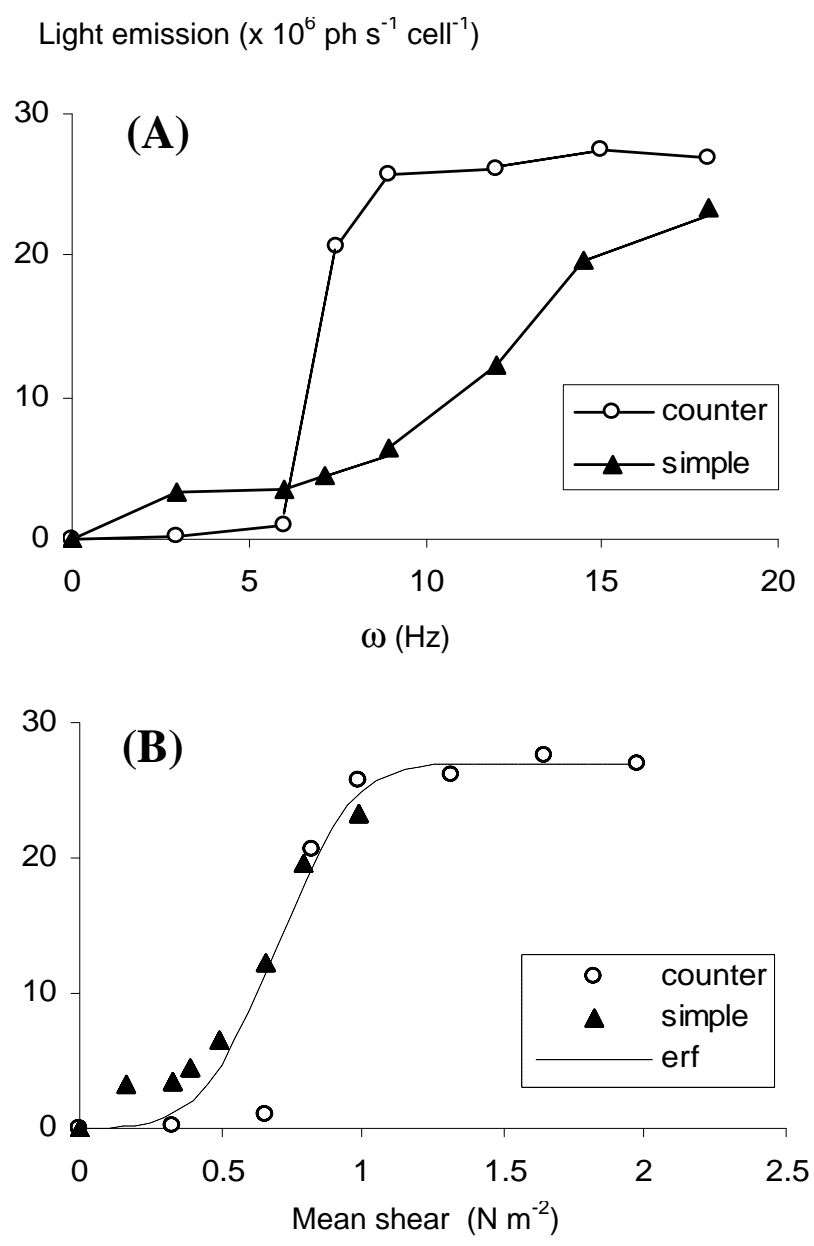

Fig. 6. Bioluminescent response for different abrupt starts at different values of final rotation frequencies. In the "simple" experiments, only the outer cylinder rotates while in the "counter" experiments, both cylinders rotate in opposite directions. The responses versus rotation frequency (A) follow " $\mathrm{S}$ " shape curves that collapse when represented as functions of the mean shear $(\mathbf{B})$. The dotted line is an error function fit of the experimental data points.

The response curve can be fitted by an error function (erf) whose parameters have been adjusted to the measurement points. Therefore, this curve represents the bioluminescence response of the Pyrocystis noctiluca cells when stimulated by an accelerated shear flow.

\section{Histogram of bioluminescent thresholds}

As seen in Fig. 6B, only a certain amount of the cells emits light when stimulated by the accelerated shear flow; the thresholds of the other cells being higher than the applied constraint. The bioluminescence response can therefore be interpreted as the cumulated histogram of thresholds under a given mechanical constraint (shear and acceleration). The distribution of these thresholds can then be calculated by the derivation of the response curve. This derivative is given in Fig. 7 together with the Gaussian probability density function

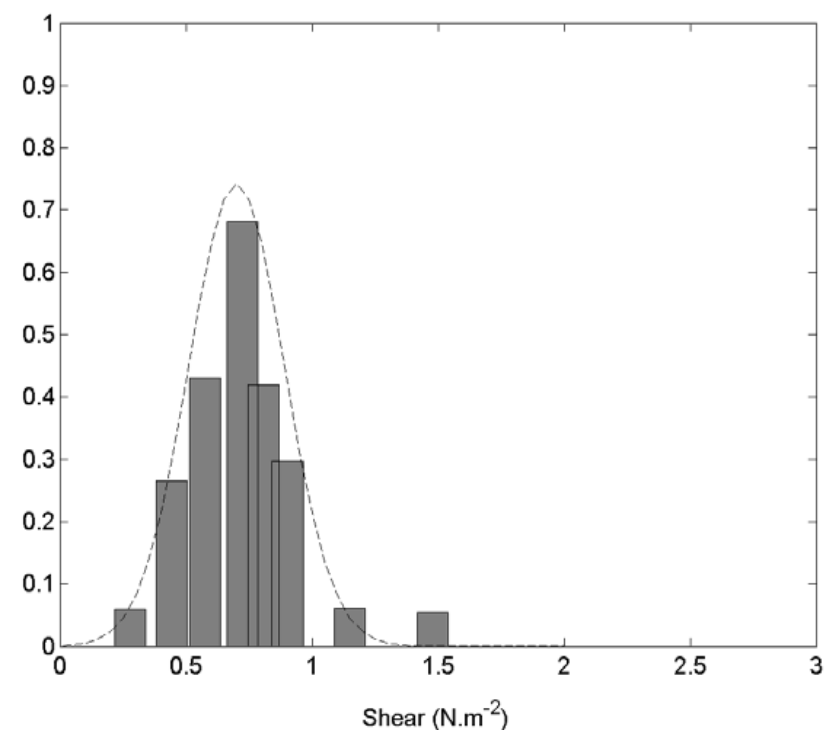

Fig. 7. Distribution of the experimental bioluminescence thresholds calculated as the derivative of the light emission response. The dotted Gaussian curve is the derivative of the error function fit while the bars represent the experimental data.

that is the derivative of the erf fitting function. The mean bioluminescent threshold is equal to $0.7 \mathrm{~N} \mathrm{~m}^{-2}$ and the standard deviation of its distribution is $0.27 \mathrm{~N} \mathrm{~m}^{-2}$. We speculate that these values are species dependent. The fact that a unique threshold is not obtained expresses the diversity of the bioluminescence response for each organism and also among the dinoflagellate population. The mean threshold value that we obtain is very closed to the one measured by Blaser et al. (2004) in different flow configurations, however it is higher than the one measured by Latz et al. (1994) in their Couette flow apparatus with Pyrocystis noctiluca and in their pipe flow experiments on Pyrocystis fusiformis (Latz et al., 2004). Note however that these apparent contradictions in the different determinations of thresholds may come from the fact that the lowest values obtained for the thresholds (around $0.1-0.3 \mathrm{~N} \mathrm{~m}^{-2}$ in Latz et al., 1994) may correspond to values on the left wing of the Gaussian distribution. The corresponding cells are indeed very sensitive to shear and any temporal changes in their environnement (inlet of a pipe, aperture, Ekman eddies, wall proximity ...) might trigger the bioluminescent reaction. In this case the disagreement between the different bioluminescence threshold determinations would only be apparent.

\section{Conclusion}

We have shown in this study the crucial role played by flow acceleration in the bioluminescence of Pyrocystis noctiluca. First of all, we have confirmed that a simple uniform and constant shear cannot trigger the main bioluminescent response in this species (the only emitted light coming from spurious finite size effects, or isolated flashes that are 
believed to come from very sensitive cells hitting the wall). In the same way, we prove in a second set of experiments that acceleration alone without shear is also inefficient. This result is consistent with the results of Latz et al. (2004). Finally the response of Pyrocystis noctiluca to accelerated shear flows is described and fitted to an erf function. The mean threshold value $\left(0.7 \mathrm{~N} \mathrm{~m}^{-2}\right)$ is in agreement with recently obtained determination $\left(0.6 \mathrm{~N} \mathrm{~m}^{-2}\right)$ by Blaser et al. (2004). The large distribution of thresholds traduces the diversity among the organism population but will unfortunately complicate a lot the use of bioluminescence as a flow diagnostic.

Edited by: F. G. Schmitt

Reviewed by: two referees

\section{References}

Blaser, S., Kurisu, F., Satoh, H., and Mino, T.: Hydromechanical stimulation of bioluminescent plankton, Luminescence. 17, 370380, 2002.

Burkholder, T.: Permeability of $\mathrm{C} 2 \mathrm{C} 12$ myotube membranes is influenced by stretch velocity, Biochim. Biophys. Res. Com., 305, 266-270, 2003.

Cadot, O., Douady, S., and Couder, Y.: Characteristics of the low pressure filaments in three-dimensional turbulent shear flow, Phys. Fluids 7, 630-646, 1995.

Couette, M.: Studies of the friction of fluids, Ann. Chim. Phys., 21, 433-510, 1890

Cussatlegras, A. S. and Le Gal, P.: Bioluminescence of the dinoflagellate Pyrocystis noctiluca induced by laminar and turbulent Couette flow, J. Exp. Mar. Biol. Ecol., 310, 227-246, 2004.

Danaila L., Le Gal, P., Anselmet, F., Plazza, F., and Pinton, J. F.: Some new features of the passive scalar mixing in a turbulent flow, Phys. Fluids, 11, 636-646, 1999.
Fogel, M. and Hastings, J. W.: Bioluminescence: Mechanism and mode of control of scintillon activity, Proc. Nat. Acad. Sci. USA, 69, 690-693, 1972.

Guillard, R. R. L. and Ryther, J. H.: Studies of marine planktonic diatoms, 1. Cyclotella nana Hustedt, and Detonula confervacea (Cleve), Gran. Can. J. Microbiol., 8, 229-239, 1962.

Latz, M. I., Case, J. F., and Gran, R.: Excitation of bioluminescence by laminar fluid shear associated with simple Couette flow, Limnol. Oceanogr., 39, 1424-1439, 1994.

Latz, M. I., Juhl, A. R., Ahmed, A. M., Elghobashi, S. E., and Rohr, J.: Hydromechanic stimulation of dinoflagellate bioluminescence: a computational and experimental study, J. Exp. Biol., 207, 1942-1951, 2004.

Latz, M. I., Nauen, J. C., and Rohr, J.: Bioluminescence response of four sepcoes of dinoflagellates to fully developed pipe flow, $\mathrm{J}$ Plankton Res., 26 (12), 1529-1546, 2004.

Rohr, J., Allen, J., Losee, J., and Latz, M. I.: The use of bioluminescence as a flow diagnostic, Phys. Lett. A, 228, 408-416, 1997.

Rohr, L., Latz, M. I., Fallon, S., Nauen, J. C., and Hendricks, E.: Experimental approaches toward interpreting dolphinsstimulated bioluminescence, J. Exp. Biol., 201, 1447-1460, 1998.

Rohr, J. J., Hyman, M., Fallon, S., Latz, M. I.: Bioluminescence flow visualization in the ocean: an initial strategy base on laboratory experiments, Deep-Sea Research I, 49, 2009-2033, 2002.

Schilichting, H.: Boundary-Layer Theory, New-York, McGrawHill, 1979.

Stokes, M. D., Deane, G. B., Latz, M. I., and Rohr, J.: Bioluminescence imaging of wave-induced turbulence, J. Geophys. Res. 109, C01004, doi:10.1029/2003JC001871, 2002.

Wang, J. H.-C., Goldschmidt-Clermont, P., Wille, J., and Yin, F.CP.: Specificity of endothelial cell reorientation in response to cyclic mechanical stretching, J. Biomechanics, 34, 1563-1572, 2001. 